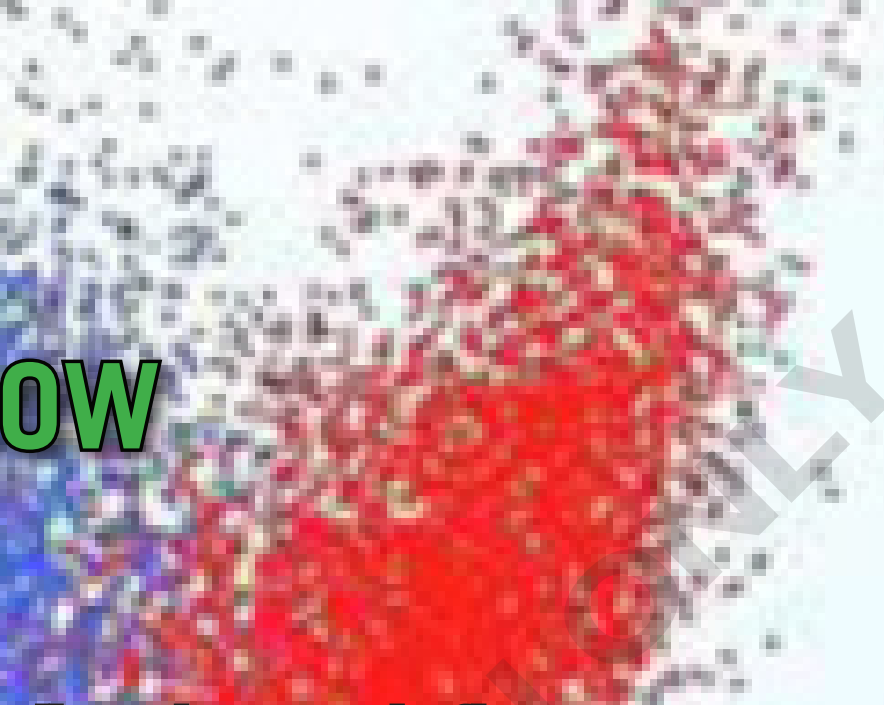

From bacteria to chroulating tumor cells, advances in flow cytometry - technollogy are pushithe the boundaries of cell blology research

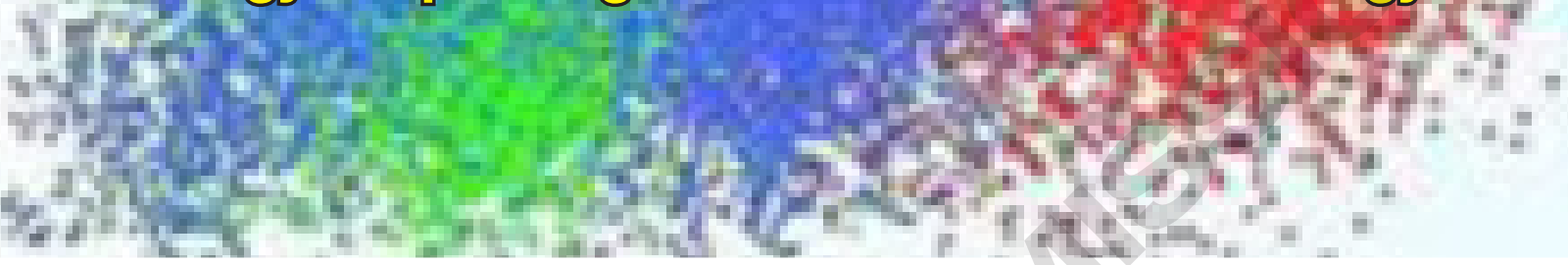

he human gut is an amazing environment containing millions upon millions of bacteria interacting with one another. We are now learning that these microscopic inhabitants also interact with our own cells in a myriad of ways. Recent studies have revealed that intestinal bacteria play essential roles in human health, and alterations in the relative abundances of different bacterial species can lead to significant health changes or even cause disease.

Understanding the ways in which these different microbes interact and the roles that they play in shaping human gut functions has become a cornerstone of many research efforts, leading to new insights in our understanding of the microbiome. But sorting through this vast array of bacteria requires technologies capable of isolating and sorting specific subpopulations based on certain characteristics. This is the reason that flow cytometry has emerged in recent years as a crucial technology platform in the field of microbiomics.

\section{Sensing a change}

As gut microbes go about their basic functions, they produce a variety of signaling molecules that are used to communicate with other bacteria and human cells. The timing of when these signaling molecules are released and exactly how other cells respond to specific microbial signaling cues are largely unanswered questions that many researchers would like to study in greater detail.
The strategy of using modified bacteria possessing engineered sensors to "eavesdrop" on communications between the inhabitants of microbial environments got its start nearly a decade ago. Recently, researchers from Rice University and Baylor University in Texas took advantage of this idea to engineer a novel strain of bacteria that could report on the intracellular levels of two key metabolites in a mouse model of colitis (1). Even though mounting data show mammalian gut function is regulated through cross-talk between host cells and resident bacteria, deciphering this cross-talk and the signaling molecules involved has proven to be quite a challenge.

The Rice and Baylor groups, led by bioengineering professor Jeffrey Tabor, were curious about the role sulfur-reducing bacteria present in the gut might be playing in the emergence of colitis. The researchers identified two sensors, one for the metabolite thiosulfate and the other for the metabolite tetrathionate, which they were then able to engineer into gut-adapted E. coli strains possessing fluorescent markers. Tabor's team was particularly interested in assessing changes in thiosulfate and tetrathionate levels since these metabolites can be used as a measure of the levels of sulfur metabolism in the gut. By attaching green fluorescent protein (GFP) to each of their engineered sensors, the researchers could measure changes in fluorescence levels as a proxy for sulfur metabolism status in the mouse gut. To quantify the numbers of the engineered fluorescent bacteria responding to the 


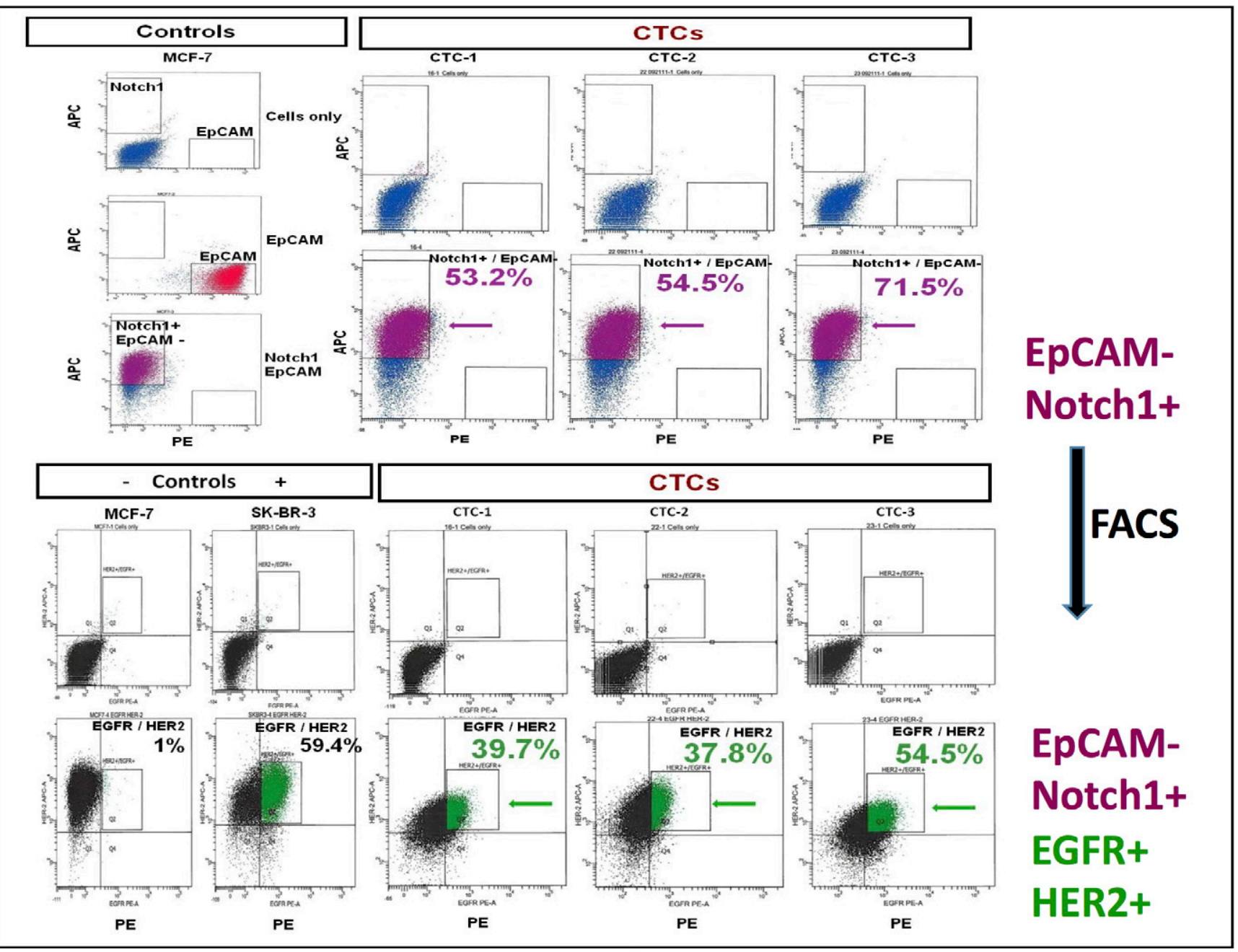

Researchers used flow cytometry to sort circulating tumor cell (CTC) populations in blood to better understand the mechanisms underlying tumor metastasis. Credit: D. Marchetti.

metabolites during the experiment, the research team turned to a flow cytometry workflow.

Flow cytometry data showed that when it came to inflammation in the colon, output from the thiosulfate sensor was elevated, implying that sulfur metabolism was indeed involved in the colitis seen in the mouse model. Based on this result, Tabor and his co-authors suggested that thiosulfate might be a useful biomarker for inflammation and colitis in the future.

In their study, Tabor and his colleagues were able to specifically engineer thiosulfate and tetrathionate bacterial sensors that could in turn be isolated using flow cytometry. But the approach they took in developing their sensors is actually more robust, and could be applied to generate other bacterial metabolite sensors in the future, opening up microbiome studies for a greater range of biological processes and phenotypes in the gut.

\section{Multiple colors, multiple differences}

Flow cytometry technology can be used to quickly detect and isolate specific cell types within a sample, especially if the cells are labeled with GFP or another suitable visual marker. But that's only the start of what flow cytometry is capable of doing these days when it comes to cell selection. In fact, many researchers and methods developers would argue that the true value of the technology lies in its multidimensional capabilities-where more colors mean more data.

The development of multi-parametric flow cytometry instrumentation and technology has proceeded at a rapid pace in recent years. Back in 1970s and early 1980s, only two fluorescence parameters could be sorted during a flow cytometry experiment. It was not until later in the 1980s, following the discovery and development of new fluorochromes along with enhancements in laser technologies and new software for 


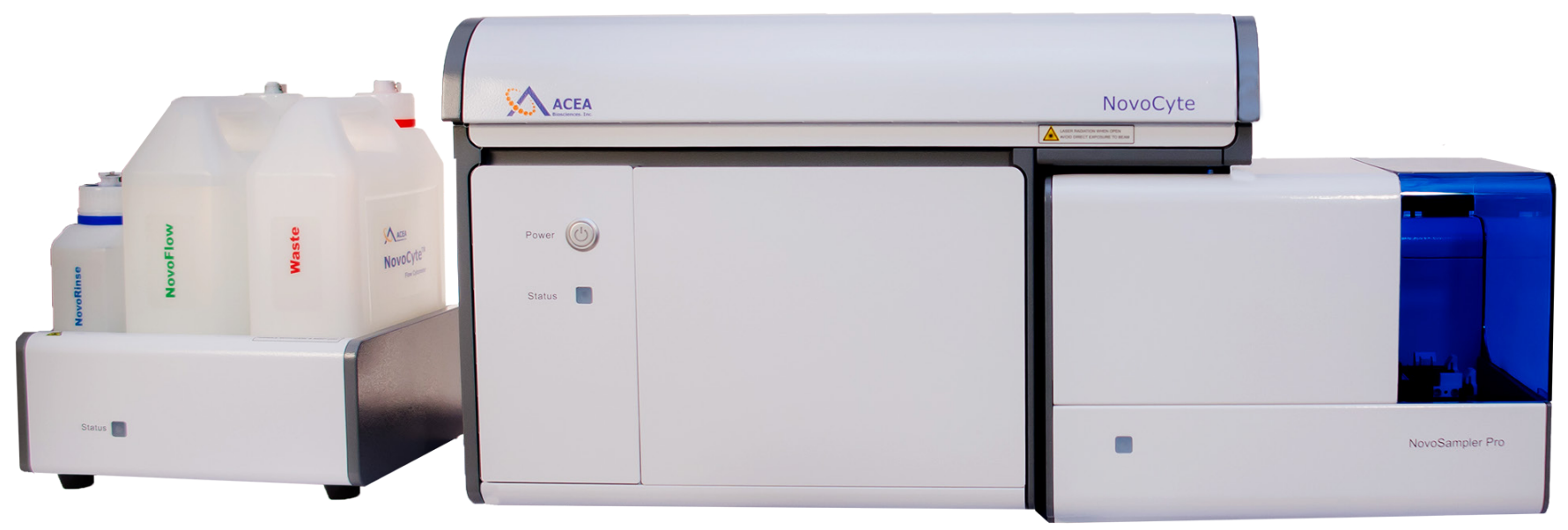

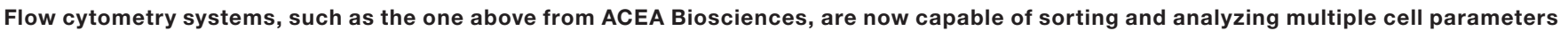
in a single experiment. Credit: ACEA Biosciences.

experimental analysis, that flow cytometry entered its next phase of evolution-a period where the number of fluorescence parameters that could be sorted during a flow cytometry experiment grew on a nearly annual basis.

Today, most commercially available flow cytometry instruments have the capacity to sort cells based on 8 to 13 different parameters, with the current state-of-theart being able to take advantage of 18 different colors for cell sorting. Moving beyond 18 colors is not possible at the moment due to the current number of different fluorophores that can be spectrally resolved by available lasers. However, developers have found some ways to get around this limitation. Mass cytometry is a technique that combines flow cytometry with mass spectrometry to analyze more than 40 parameters in a single experiment without the need for fluorophores. Still, even given the current color limits, these latest advances in flow cytometry technology have made it possible for researchers to isolate and study very specific cell types, and even subsets of cell populations, to glean unique information on cell composition and abundance for fields as diverse as immunology and cancer research.

\section{Singling out cells}

Circulating tumor cells (CTCs) are found in the bloodstream and have been implicated to play roles as "seeds" of fatal cancer metastasis. As such, identifying these cells and understanding their roles in metastatic onset could prove critical for detecting the presence of cancer as well as for monitoring disease progression in those patients being treated with specific therapies.

In 2015, Dario Marchetti from the Houston Methodist Research Institute in Texas and his colleagues reported on the use of flow cytometry to isolate CTC subsets possessing properties related to breast cancer dormancy (2). The idea was to compare CTC populations from patients with breast cancer metastasis to those without metastasis in an effort to identify difference in their circulating tumor cell profiles. The use of multi-parametric flow cytometry made it possible for Marchetti's team to identify different CTC cells by their unique phenotypic properties, including adhesion, proliferation, and invasion, using reagents that targeted the expression of specific cell-surface markers.

"Multi-parametric flow cytometry offered us an excellent tool with which to both isolate and interrogate CTC populations," explains Marchetti, who also directs the Biomarker Research Program Center at Houston Methodist. It's important to note that developing robust assays to interrogate numerous cellular parameters during a flow cytometry experiment requires time and effort for optimization. But it turns out that Marchetti's lab has actually been using flow cytometry to explore CTC biology for a number of years now. "Flow cytometry experiments have allowed us to isolate rare cells, including CTCs, that often cannot be captured with the same high level of rigor using other methods."

\section{CTC discoveries}

Two years earlier, Marchetti's lab was already applying flow cytometry in 
cancer studies. At the time, it was known that breast cancer was one of the most common cancers to metastasize to the brain, and Marchetti wanted to understand the mechanisms causing this outcome.

Applying a multi-parametric flow cytometry approach, Marchetti's group uncovered CTCs in patients that were not expressing the common carcinoma epithelial cell adhesion molecule (these are classified as EpCAM-negative cells) and possessed a gene signature important for brain cancer metastasis (3). Further work revealed that these cells promoted breast cancer brain metastasis when injected in xenografts.

Marchetti suspected that most of the other commercially available approaches being used at the time to isolate and characterize CTCs would not have been able to capture specific CTC subpopulations found to be associated with brain cancer brain metastasis.

"We needed alternative methods to the established approaches for CTC enumeration, such as CellSearch, and we realized then that a flow cytometry workflow would provide the ideal solution here," Marchetti recalls.

Spurred by their EpCAM-negative CTC capture, Marchetti's team became increasingly curious about the roles that other CTCs might be playing in breast cancer metastasis, cells that he refers to as "flying under the radar" of detection for many technologies in the way that EpCAM-negative CTCs had previously.

It's critical to note that CTCs are exceedingly rare in blood samples, making their isolation that much more challenging. Add to this the fact that Marchetti was now focused on further subdividing CTCs by tumor origin and proliferative capacity, and it's clear he needed to develop a robust multi-parametric flow cytometry strategy for narrowing down any CTC associations with breast cancer brain metastasis.

For his most recent study on breast cancer, Marchetti employed a multistep approach for hunting unique CTCs. First, the team isolated CTCs based on EpCAM agnostic status and epithelial versus stemcell-like properties. From there, a combination of six different surface expression markers were assayed to classify the functions of the various CTC subsets found. Finally, isolated CTC subsets were investigated to determine gene expression profiles and biomarker pathways, similar to how EpCAM-negative CTCs had been studied 2 years earlier. When all was done, Marchetti's team was able to use the data from their CTC isolation and characterization efforts to start dissecting apart the molecular pathways and mechanisms involved in CTC brain organ-homing and, thus, better understand breast cancer CTC characteristics in relation to brain metastasis onset (i.e., detectable by MRI as accepted

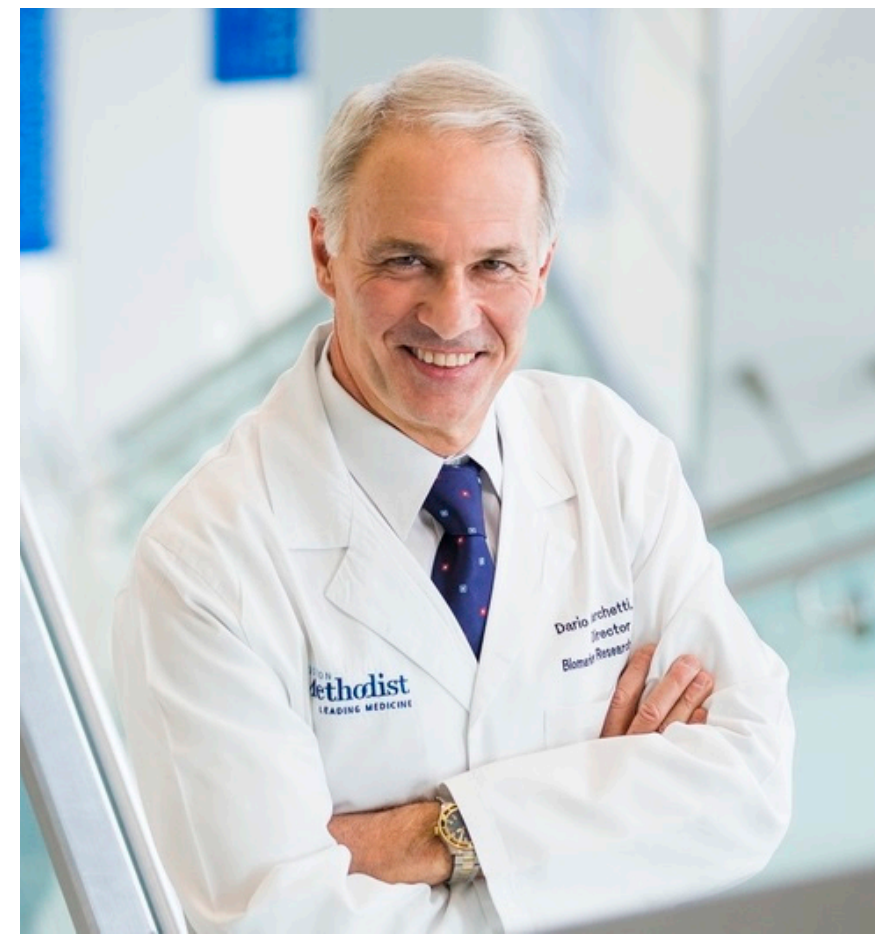

Dario Marchetti from Houston Methodist Research Institute has been using flow cytometry in his breast cancer research studies. Credit: D. Marchetti.

standard of care compared to tumor progression). The next step, according to Marchetti, will be to identify biomarkers associated with breast cancer CTCs that can be used in the detection and monitoring of cancer progression along with therapy decisions and evaluation of therapy efficacy.

In many ways, flow cytometry can be thought of as a re-emerging technology. While the basic technique is well-established, its use in the lab today is moving beyond simply separating and sorting cell populations. And as flow cytometry technology continues to advance in the years to come, with more researchers seeking to dissect cell populations and their interactions, our understanding of the cellular world will only continue to grow.

\section{References}

1. Daeffler, K. et al. 2017. Engineering bacterial thiosulfate and tetrathionate sensors for detecting gut inflammation. Molecular Systems Biology. 13:923.

2. Vishoi, M. et al. 2015. The isolation and characterization of CTC subsets related to breast cancer dormancy. Scientific Reports. 5:17533.

3. Zhang, L. et al. 2013. The identification and characterization of breast cancer CTCs competent for brain metastasis. Science Translational Medicine. 5:180ra48.

Written by Nathan Blow, Ph.D. 㖥

BioTechniques 62: 201-205 May 2017

doi: 10.2144/000114543 